\title{
Prospective randomized controlled trial for patch augmentation in rotator cuff repair: 24-month outcomes
}

\author{
Paolo Avanzi, MD ${ }^{a}$, Luca dei Giudici, MD ${ }^{b}$, Antonio Capone, MD, PhD ${ }^{c}$, \\ Gaia Cardoni, MD ${ }^{c}$, , Gianluigi Lunardi, PharmD ${ }^{d}$, Giovanni Foti, MD ${ }^{e}$, \\ Claudio Zorzi, MD
}

\author{
${ }^{a}$ Department of Orthopaedics, Sacro Cuore-Don Calabria Hospital, Negrar, Verona, Italy \\ ${ }^{b}$ Orthopaedic Unit, Casa di Cura "Villa Igea" Hospital, Ancona, Italy \\ ${ }^{c}$ Department of Surgical Science, Orthopaedic Clinic, University of Cagliari, Ospedale Marino, Cagliari, Italy \\ ${ }^{d}$ Department of Medical Oncology, Sacro Cuore-Don Calabria Hospital, Negrar, Verona, Italy \\ ${ }^{e}$ Department of Radiology, Sacro Cuore-Don Calabria Hospital, Negrar, Verona, Italy
}

\begin{abstract}
Background: To evaluate the anatomic integrity of rotator cuff repair performed by medialized single row and augmented by a porcine dermal patch, in comparison with a nonaugmented group.

Methods: We conducted a single-center, prospective, double-blinded, randomized controlled trial. The sample size was predefined, and patients were divided into a study group and a control group, assessed preoperatively and at 1, 3, 6, 12, and 24 months. The EuroQol-visual analog scale; Constant-Murley questionnaire; Disabilities of the Arm, Shoulder and Hand Score; and Simple Shoulder Test were administered. The humeral-acromial distance was calculated on radiographs. Tendon thickness, tear extension, and tendon signal intensity were all measured on magnetic resonance images (MRIs) along with an evaluation of footprint extension and a classification into one of 4 healing grades-healed, thinned, partially healed, not healed.

Results: The study population consisted of 92 patients who were equally randomized into 2 homogenous groups. Sixty-nine patients completed the 24-month follow-up. The study group showed a healing rate of $97.6 \%$ compared with $59.5 \%$ for the standard repair group. The study group showed better results in terms of repaired tendon thickness and footprint coverage, with a $P$ value $<.05$, although the tendon density was comparable. The study group showed better strength recovery and functionality with the outcome scores submitted. During the entire study, only 2 patients reported complications, calling for a biopsy during revision surgery.

Conclusions: Rotator cuff repairs augmented with a porcine dermal patch resulted in excellent clinical outcomes with a higher healing rate and close-to-normal MRI findings. The technique is safe and effective; in addition, it is reproducible and allows for better outcomes compared with those of standard medialized single-row repairs.
\end{abstract}

This study was approved by the Comitato Etico della Provincia di Verona (study no. 394 CEP).
*Reprint requests: Gaia Cardoni, MD, Department of Surgical Science, Orthopaedic Clinic, University of Cagliari, Ospedale Marino, Lungomare Poetto 12, 09126 Cagliari, Italy.

E-mail address: gaia.cardoni@gmail.com (G. Cardoni). 
Level of evidence: Level II; Randomized Controlled Trial; Treatment Study

(C) 2019 Journal of Shoulder and Elbow Surgery Board of Trustees. All rights reserved.

Keywords: Rotator cuff repair; shoulder augmentation; porcine dermal patch; shoulder arthroscopy; shoulder controlled trial; rotator cuff augmentation

Degenerative rotator cuff tears are common in middleaged patients, with a prevalence up to $40 \%^{15}$ and result in pain and dysfunction in the majority of cases. ${ }^{32}$ The gold standard for treatment is surgical repair after the failure of conservative management, but retear rates can range from $34 \%$ up to $94 \%,{ }^{21}$ despite pain relief. Retears result mainly from 2 factors: tension overload of the surgical suture and insufficient biological healing. Complete footprint coverage is sought to prevent a failure ${ }^{8}$ to improve healing at the tendon-to-bone interface, to improve mechanical factors, and to obtain a more anatomic repair. It was demonstrated that incompletely covered repairs might result in less than optimal healing, ${ }^{33}$ whereas maximizing the contact area and contact pressure between the tendon and tuberosity might enhance biological healing, strength, and tendon function. ${ }^{30}$ Moreover, Burkhart et $\mathrm{al}^{6}$ demonstrated how a gap of at least $5 \mathrm{~mm}$ between the repaired tendon and its footprint has a low chance of healing and that this gap most probably is a consequence of the low-level loading of the rotator cuff repair resulting from routine muscle contraction, which occurs even in a postoperative sling.

The aim of the augmentation of rotator cuff repair is to mechanically offload the tendon repair; this technique has recently demonstrated promising results in terms of retear rates. ${ }^{3}$ The rationale behind these procedures is the use of a scaffold, which can be synthetic, biosynthetic, or biological (crosslinked, and terminally sterilized), ${ }^{28}$ to mechanically protect the repair during the immediate postoperative time so as to enhance the speed and/or the quality of the healing. ${ }^{26}$ Synthetic scaffolds hold increasing interest because of good clinical outcomes associated with their use, but several concerns exist, such as a limited tissue ingrowth potential, a possible acute inflammatory response, chronic inflammation, and issues related to foreign material reactions; whereas biological scaffold are providing convincing pieces of evidence, as no sign of foreign body reaction or chronic inflammation are reported in the literature. ${ }^{20}$ The addition of such grafts has been found to reduce gap formation from $40 \%$ to $3 \%$ at the tendon-bone repair site, markedly increasing the load necessary to produce the critical gap of $5 \mathrm{~mm}$, and support a mean of $35 \%$ of the global load applied to the repair. ${ }^{27}$ A porcine dermal matrix (Conexa; Tornier Inc, Edina, MN, USA), produced by decellularization through high-salt and detergent processes while maintaining the architecture and biochemistry of the dermis, ${ }^{31}$ shows repopulation and revascularization with a minimal inflammatory response elicited by the host in animal models and a propensity to remodel to a fascialike architecture by 6 months. ${ }^{10}$

\section{Purpose and hypothesis}

The purpose of this study was to evaluate the anatomic integrity of rotator cuff repair performed by medialized single row and augmented by a porcine dermal patch, in comparison with a nonaugmented group. The primary hypothesis was that a better outcome, defined as tendon-tobone healing, could be achieved in the biologically augmented group. The secondary hypothesis was that a better quality of the repaired tendon in the augmented group at the magnetic resonance image (MRI) evaluation, with a thicker tendon, a wider footprint extension, and a greater physiologic signal intensity, as well as a faster strength recovery and better outcome scores, could be achieved.

\section{Materials and methods}

This study is a single-center, prospective, double-blind, randomized controlled trial with 2 parallel groups and was approved by the institutional ethical committee (IEC number: 2013/3) in accordance with the Declaration of Helsinki. Screening for patients started on December 1, 2014.

Inclusion criteria were age between 50 and 80 years; presence of degenerative small and medium rotator cuff tears; superior, anterosuperior, and posterosuperior tears; Ellman-Patte retraction index $^{24}$ type 1 and 2 (early and late); Fuchs muscular index ${ }^{12}$ type negative and neutral; Goutallier fatty infiltration index ${ }^{13}$ type 0,1 , and 2 ; and osteoarthritis grade 0 or 1 according to Fukuda. ${ }^{14}$

Exclusion criteria were post-traumatic rotator cuff tears, concomitant shoulder pathologies, previous shoulder surgeries, corticosteroids injections in the 6 months preceding study enrollment, disease of the connective tissue, infective and rheumatoid diseases, and major systemic conditions.

Degenerative rotator cuff tears were diagnosed through referred symptoms history, clinical examination, radiographs (4 views: anteroposterior neutral rotation, anteroposterior internal rotation, arch view, and axillary view), and high-field MRI (1.5 T) with 3-mm slices.

All patients eligible for enrollment signed a specific informed consent at the time of surgical indication, and we recorded all the demographic and preoperative data for each patient. 

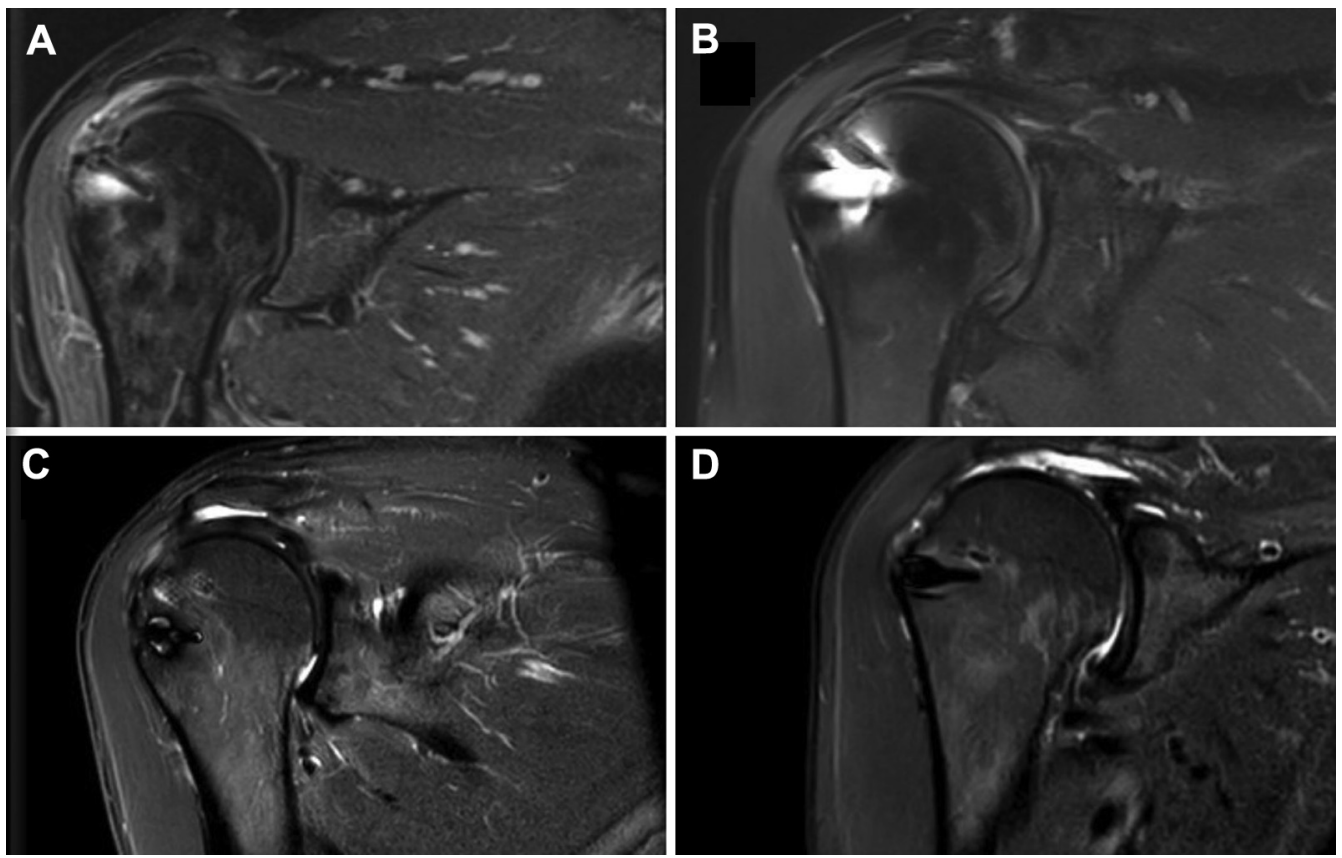

Figure 1 Healing categories: (A) healed; (B) healed but thinned; (C) partially healed; (D) retear.

Clinical assessment was performed using the EuroQol-visual analog scale pain score, the Constant-Murley questionnaire, the Disabilities of the Arm, Shoulder and Hand score, and the Simple Shoulder Test score. Humeral-acromial distance was calculated on radiographs; tendon thickness (calculated at $10 \mathrm{~mm}$ from the tendon edge), tear extension (medial to lateral, and anterior to posterior), and tendon signal intensity (evaluated $5 \mathrm{~mm}$ lateral to the tendon edge, in T2-weighted sequence, as follows: healthy tendon is black with 0 points in value, tear is white with 500 points in value) were measured on MRI as well as Ellman-Patte tendon retraction, Goutallier fatty infiltration, and Fuchs muscular index. At each follow-up, the MRI assessment, performed by an independent observer, also included an evaluation of the footprint extension from medial to lateral (from the cartilage margin to the greater tuberosity) and a classification of the repair into one of the following 4 categories: healed, thinned but healed, partially healed, or not healed (Fig. 1). We considered the first 2 categories as positive outcomes because there is no interruption in the tendon continuity, and the latter 2, partially healed and not healed, as adverse outcomes. We performed the measurements of the tendon thickness and of the footprint with the DICOM reader program that measures up to 1/100th of a millimeter; to reduce errors, we reported the measure up to $1 / 10$ th of a millimeter.

A sample size analysis was performed using a Pearson $\chi^{2}$ test for a 2-sample proportion, verifying the efficacy of the patch with a healing proportion of $40 \%$ in group B (no patch) vs. $70 \%$ in group A (with patch), with an alpha of 0.05 and a beta equal to 0.80 , which called for 46 patients in each group, considering a dropout rate of $10 \%$.

Randomization was carried out with a closed envelopes system, to be opened during surgery by a surgeon blinded to clinical and radiologic evaluations.
Surgery was standardized: a shoulder arthroscopy was performed in lateral decubitus position by the same surgeon; rotator cuff repair was obtained with a single-row technique using 2 PEEK anchors loaded with 2 sutures each (Insite; Tornier Inc). At this stage of the surgery, a nurse opened the sealed envelope, and the patient was randomized into one of the 2 groups: group A, which would receive the augmentation of a porcine dermal patch (Conexa; Tornier Inc), and group B, which would not receive the augmentation and which would serve as the control group. In both groups, a biceps tenotomy and a subacromial decompression ("cutting block" technique) were performed.

In group A, the patch was applied according to the "goal post" technique. ${ }^{1}$ After sizing with an arthroscopic ruler and appropriate trimming, the patch was fixed medially with 2 simple stitches of high-strength sutures at the myotendinous junction; laterally, it was fixed over and lateral to the footprint on the major tuberosity with a transosseous equivalent technique with crossing sutures loaded onto 2 metal knotless anchors (Fig. 2) (Piton; Tornier Inc).

A 4-month-long rehabilitation period was standardized for both groups and included a first phase focused on suture protection, a second phase focused on passive range of motion recovery, and a third phase focused on strength recovery and reconditioning.

All patients were followed up at 1, 3, 6, 12, and 24 months after surgery, with the same clinical and radiologic assessment that was performed at the time of enrollment. Clinical assessment was performed at 3, 6, 12, and 24 months, and MRI evaluations were performed at $1,6,12$, and 24 months after surgery.

During the whole study, blinding was achieved by having each investigator strictly cover a specific role: enrollment and preoperative data gathering, surgery, clinical and radiologic examination, and administration of outcome scores at follow-ups. 

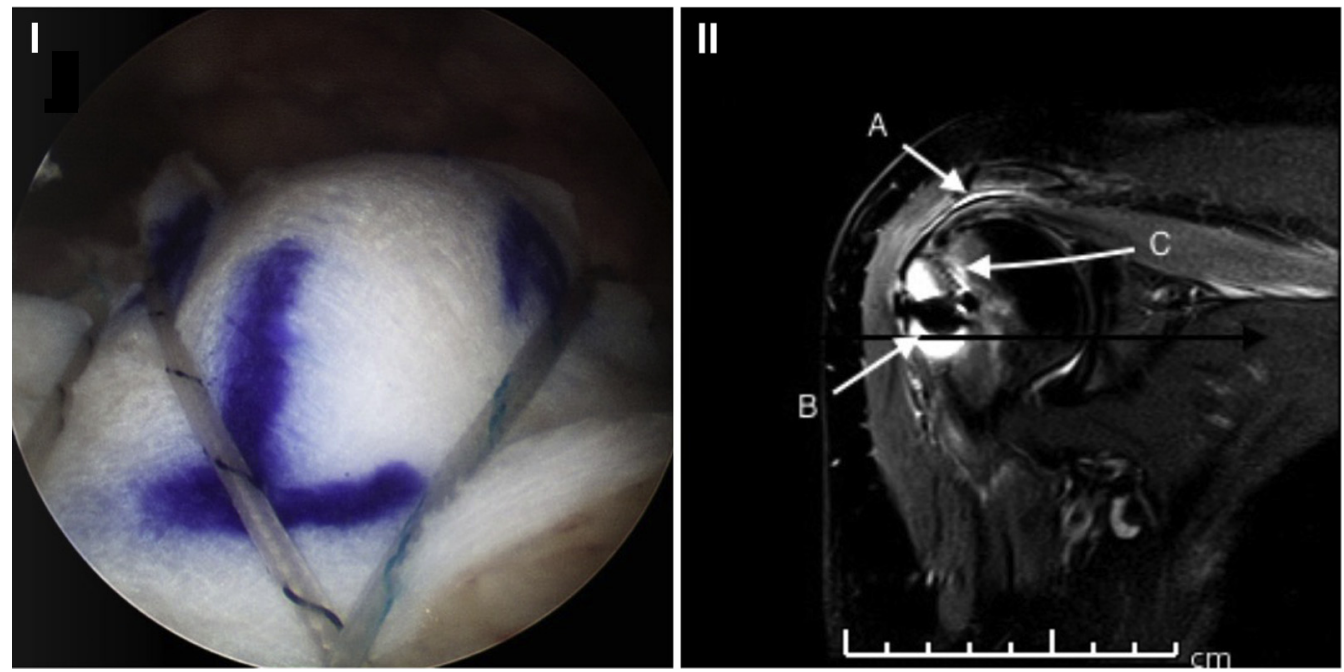

Figure 2 Goalpost technique. (I), intraoperative image of the repair construct according to the goal post technique, from the lateral portal. It can appear as a double-row-like repair, with the scaffold interposed between the repaired tendon (inferiorly) and the anchor sutures (superiorly); (II), 1-month postoperative magnetic resonance images; $A$, membrane; $B$, lateral suture row; $C$, medial suture row.

Statistical analysis was performed using Stata, version 14.0 SE (Stata Corp, College Station, TX, USA). The $\chi^{2}$ test was used to evaluate associations between categorical variables. MannWhitney (unpaired data) or Wilcoxon (paired data) tests were used to compare continuous variables between groups. A 2 -sided $P$ value of less than .05 was considered to be statistically significant.

\section{Results}

We enrolled 92 patients, randomized into 2 groups; group A included 46 patients treated with a medialized single-row repair augmented with a porcine dermal patch, and group B included 46 patients treated with a standard medialized single-row repair.
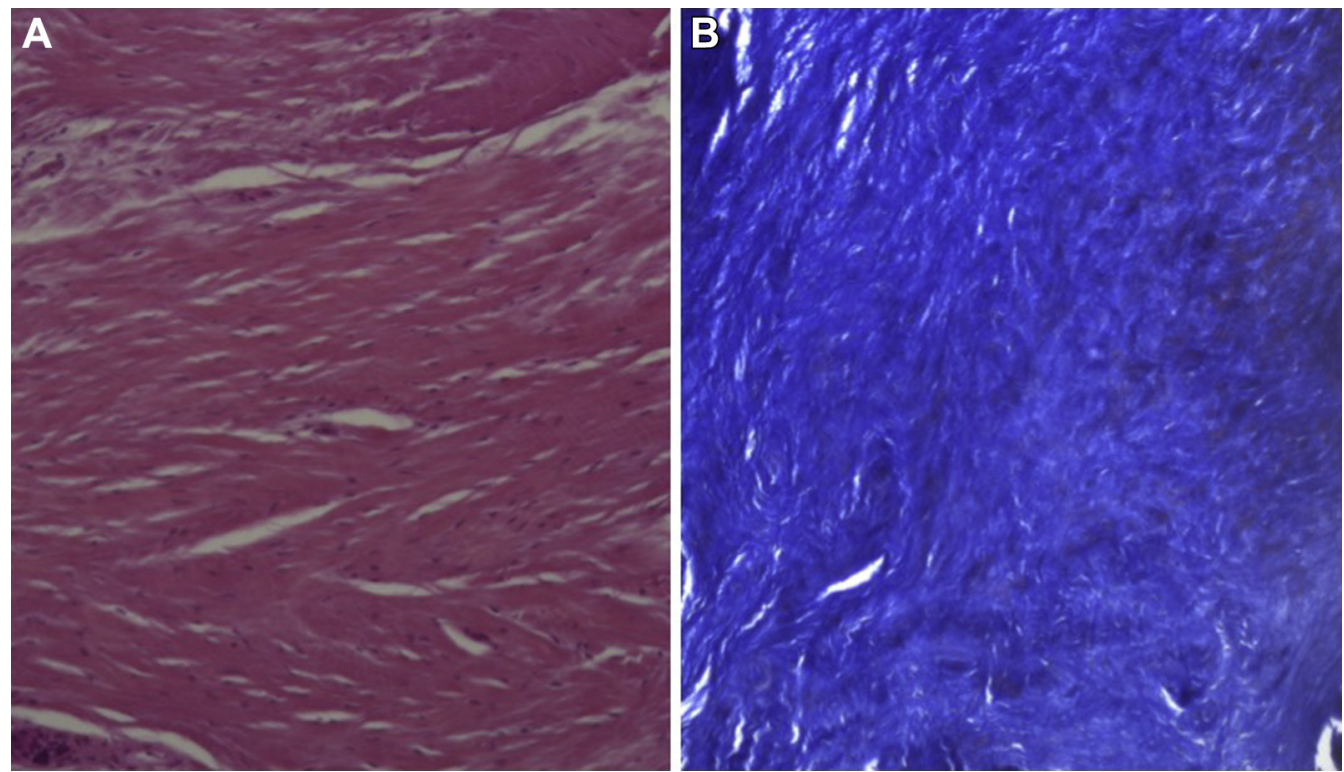

Figure 3 Biopsy sample from a second look of a patient who suffered a traumatic mobilization of a lateral anchor. The tissue is characterized by quite homogenously distributed fibroblastlike cells, scattered areas of neoangiogenesis with a few perivascular inflammatory cells, and minimal spots of myxoid degeneration and adipose degeneration. No chondral metaplasia, macrophages, or giant cells are evident (A, hematoxylin and eosin staining, $\times 100$; B, Masson's Trichromic staining, $\times 100$ ). 
Table I Patient demographics

\begin{tabular}{|c|c|c|c|}
\hline & $\begin{array}{l}\text { Group A } \\
(n=46)\end{array}$ & $\begin{array}{l}\text { Group B } \\
(n=46)\end{array}$ & $P$ value \\
\hline \multicolumn{4}{|l|}{ Sex } \\
\hline Female & 32 & 24 & \\
\hline Male & 14 & 22 & .09 \\
\hline Median age (range), yr & $68(58-78)$ & $66(54-76)$ & .06 \\
\hline Side & & & 1 \\
\hline Left & 14 & 14 & \\
\hline Right & 32 & 32 & \\
\hline Location & & & .48 \\
\hline Superior & 28 & 29 & \\
\hline Posterosuperior & 13 & 11 & \\
\hline $\begin{array}{l}\text { Superior to } \\
\text { anterosuperior }\end{array}$ & 2 & 5 & \\
\hline $\begin{array}{l}\text { Superior to } \\
\text { posterosuperior }\end{array}$ & 3 & 1 & \\
\hline Shape & & & .14 \\
\hline Crescent & 27 & 24 & \\
\hline V & 4 & 0 & \\
\hline U & 10 & 12 & \\
\hline Posterior L & 0 & 1 & \\
\hline Anterior L & 4 & 9 & \\
\hline Unknown & 1 & 0 & \\
\hline Type & & & .31 \\
\hline Partial & 1 & 0 & \\
\hline Complete & 45 & 46 & \\
\hline Ellmann-Patte & & & .06 \\
\hline Early & 32 & 23 & \\
\hline Late & 14 & 23 & \\
\hline Fuchs & & & .60 \\
\hline Positive & 27 & 27 & \\
\hline Neutral & 18 & 19 & \\
\hline Negative & 1 & 0 & \\
\hline Goutallier & & & .83 \\
\hline 0 & 17 & 15 & \\
\hline 1 & 22 & 22 & \\
\hline 2 & 7 & 9 & \\
\hline
\end{tabular}

The population included 36 male and 56 female patients, mean age of 67 years (group A 68 and group B 66 years). In both groups, 14 left shoulders and 32 right shoulders were treated. For each patient, we collected demographic information and clinical features in a database, evaluating the baseline MRI such as the location, shape, type, and extension of the lesion. For each lesion, we recorded the tendon retraction, muscle fatty infiltration, and muscle atrophy using the Ellmann-Patte, Goutallier, and Fuchs classification, respectively. At the baseline MRI, there were no differences between the 2 groups.

No differences are reported even in the results of the preoperative clinical assessment performed using the
Simple Shoulder Test, Disabilities of the Arm, Shoulder and Hand Score, Constant, and the EuroQol-visual analog scale scores (Table I).

During follow-up, we lost 23 patients, 2 at the 1-year follow-up ( 1 healed of the control group and 1 not healed of the experimental group) and 21 at the 2-year follow-up: 14 in group B (8 healed, 2 thinned, and 4 not healed) and 7 in group A (1 not healed, 4 healed, and 2 thinned).

At the 2-year follow-up, 38 patients in group A and 30 patients in group B were evaluated both clinically and with a new MRI.

We decided to consider the patient lost after the 1-year follow-up who was classified with an adverse outcome at the last MRI as not healed even at the 2-year follow-up; this included 4 not healed patients ( 1 in group A and 3 in group B). They were not taken into account in the clinical evaluation because the data were missing.

At the 2-year follow-up, group A included 32 healed, 8 thinned, and 1 not healed patients whereas group B included 9 healed, 13 thinned, 3 partially healed, and 12 not healed patients (Table II).

At 2 years, the healing rate was $97.6 \%$ for the augmented group, compared with $59.6 \%$ for the control group-a statistically significant difference $(P$ value $<.001)$. The difference remains statistically significant even when considering as a positive outcome only the tendons classified as healed, with a healing rate of $78 \%$ (32) for group A and $24.3 \%$ (9) for group B, with a $P$ value $<.001$ (Table II).

We wanted to understand if preoperative tendon retraction could have influenced the outcome in both groups. Considering only the early retracted tendons, group A included 28 patients who were healed in 23 cases and thinned in the remaining 5, with no adverse outcomes (Table II), showing a healing rate of $100 \%$. On the other hand, group B included 19 patients, 6 of whom were healed, 7 thinned, and 4 not healed, with a healing rate of $64 \%$. This difference was statistically significant, with a $P$ value of .003 . The difference remained statistically significant even when considering only the healed patients, with a $P$ value of .004 and a healing rate of $82 \%$ (23) for group A vs. $31.6 \%$ (6) for group B (Table II).

Considering the tendons classified as late and too late, group A (13) had a healing rate of $92.3 \%$ (12) that decreased to $69.2 \%$ (9) considering only the healed patient vs. the $50 \%$ (9) of group B (18) that decreased to $16.7 \%$ (3) considering only the healed patient; both the differences are statistically significant with $P$ values of, respectively, .015 and .004 (Table II).

Clinical scores were obtained preoperatively and postoperatively. The EuroQol-visual analog scale score decreased from 6.5 to 0.4 in group A and from 5.4 to 1.1 in group B. The Disabilities of the Arm, Shoulder and Hand Score lowered from 51.0 to 5.4 in group A and from 47.7 to 7.2 in the control group. The Constant score increased from 46.2 to 95.5 in the augmented group and from 49.8 to 92.6 in the nonaugmented group. The Simple Shoulder Test 
Table II Primary (radiologic) outcomes of the 2 groups

\begin{tabular}{lcc}
\hline & Group A (\%) & Group B (\%) \\
\hline All patients & $\mathrm{n}=41$ & $\mathrm{n}=37$ \\
Healed & $32(78.0)$ & $9(24.3)$ \\
Thinned but healed & $8(19.5)$ & $13(35.1)$ \\
Partially healed & $0(0.0)$ & $3(8.1)$ \\
Not healed & $1(2.4)$ & $12(32.4)$ \\
Early & $\mathrm{n}=28$ & $\mathrm{n}=19$ \\
Healed & $23(82.1)$ & $6(31.6)$ \\
Thinned but healed & $5(17.9)$ & $7(36.8)$ \\
Partially healed & $0(0.0)$ & $2(10.5)$ \\
Not healed & $0(0.0)$ & $4(21.1)$ \\
Late & $\mathrm{n}=13$ & $\mathrm{n}=18$ \\
Healed & $9(69.2)$ & $3(16.7)$ \\
Thinned but healed & $3(23.1)$ & $6(33.3)$ \\
Partially healed & $0(0.0)$ & $1(5.6)$ \\
Not healed & $1(7.7)$ & $8(44.4)$ \\
\hline
\end{tabular}

Left column: outcome category; central column: augmented group (A), right column: standard repair (B). First row head shows generalized outcomes, middle row head shows outcomes of patients with an Ellmann-Patte "early" retraction grade, and inferior row head shows outcomes for patients with an Ellman-Patte "late" retraction grade. Results reported are in absolute values and percentages, obtained at the latest follow-up (2 years postoperative).

score increased from 2.4 to 10.4 in the experimental group and from 2.9 to 10.4 in the control group. All differences between the pre- and postoperative scores are statistically significant, but the clinical scores were not different between the experimental and the control groups (Table III).

Density, thickness, and footprint coverage were measured on the MRI of the healed tendon at each followup to evaluate the quality of the tendon and to understand how the patch can improve it.

Footprint coverage in group A had a mean value of 9.5 mm compared with $6.1 \mathrm{~mm}$ of group B; this difference is statistically significant, with a $P$ value of .0002 . Tendon thickness is significantly higher in group A, $4.4 \mathrm{~mm}$, compared with $3.6 \mathrm{~mm}$ of group $\mathrm{B}(P=.012)$; on the other hand, tendon density is comparable between the 2 groups, with 87.3 points in the augmented group and 88.4 points in the nonaugmented group $(P=.77)$.

Tendon thickness at 1 month was found to be significantly higher than that in the control group, and even when compared with the thickness of the tendon before the repair, this is probably because of the presence of the membrane itself. Throughout the 2 years' follow-up, the thickness slightly decreased in both groups. However, in the augmented group, the mean value remained higher and was comparable to the preoperative tendon thickness whereas in the control group at 2 years it was significantly lower (Table IV).

Strength assessment revealed no difference between the 2 groups at the 2-year follow-up, with a mean of $10.5 \mathrm{~kg}$ in
Table III Secondary (functional) outcomes of the 2 groups

\begin{tabular}{|c|c|c|c|}
\hline & Group A & Group B & $P$ value \\
\hline \multicolumn{4}{|l|}{ EQ-VAS score } \\
\hline Baseline & 64.7 (21.2) & $54.4(21.8)$ & ns \\
\hline $3 \mathrm{mo}$ & $16.4(15.8)$ & $16.9(15.2)$ & ns \\
\hline $6 \mathrm{mo}$ & $7.3(8.0)$ & $6.8(8.1)$ & ns \\
\hline $12 \mathrm{mo}$ & $7.1(8.8)$ & $5.2(6.7)$ & ns \\
\hline $24 \mathrm{mo}$ & $4.0(7.7)$ & $10.6(17.8)$ & ns \\
\hline \multicolumn{4}{|l|}{ DASH score } \\
\hline Baseline & $51.0(17.1)$ & $47.7(16.6)$ & ns \\
\hline $3 \mathrm{mo}$ & $20.2(13.5)$ & $22.1(14.1)$ & ns \\
\hline $6 \mathrm{mo}$ & $8.1(10.8)$ & $9.6(9.7)$ & ns \\
\hline $12 \mathrm{mo}$ & $8.1(10.8)$ & $5.8(6.7)$ & ns \\
\hline $24 \mathrm{mo}$ & $5.4(7.6)$ & 7.2 (12.7) & ns \\
\hline \multicolumn{4}{|l|}{ SST score } \\
\hline Baseline & $2.6(2.9)$ & $2.9(2.5)$ & ns \\
\hline $3 \mathrm{mo}$ & $7.3(2.8)$ & $6.9(2.7)$ & ns \\
\hline $6 \mathrm{mo}$ & $10.0(2.2)$ & $9.7(2.5)$ & ns \\
\hline $12 \mathrm{mo}$ & $9.7(2.62)$ & $10.3(1.9)$ & ns \\
\hline $24 \mathrm{mo}$ & $10.4(2.2)$ & $10.4(2.0)$ & ns \\
\hline \multicolumn{4}{|c|}{ Constant score } \\
\hline Baseline & $46.2(14.4)$ & $49.8(11.8)$ & ns \\
\hline $3 \mathrm{mo}$ & $71.0(12.0)$ & $65.0(11.6)$ & .01 \\
\hline $6 \mathrm{mo}$ & $91.5(8.7)$ & $87.4(9.7)$ & .02 \\
\hline $12 \mathrm{mo}$ & $92.6(8.5)$ & $89.2(9.6)$ & .02 \\
\hline $24 \mathrm{mo}$ & $95.5(5.5)$ & $92.6(9.3)$ & ns \\
\hline \multicolumn{4}{|l|}{ Strength } \\
\hline Baseline & $5.6(1.8)$ & $6.1(1.9)$ & .17 \\
\hline $3 \mathrm{mo}$ & $9.2(1.4)$ & 7.7 (1.9) & $<.001$ \\
\hline $6 \mathrm{mo}$ & $10.9(1.1)$ & $9.1(1.9)$ & $<.001$ \\
\hline $12 \mathrm{mo}$ & $10.6(0.9)$ & $8.9(2.0)$ & $<.001$ \\
\hline $24 \mathrm{mo}$ & $10.5(1.1)$ & $9.6(2.2)$ & .06 \\
\hline
\end{tabular}

EQ-VAS, EuroQol-visual analog scale; DASH, Disabilities of the Arm, Shoulder and Hand; SST, Simple Shoulder Test; ns, nonsignificant. Left column indicates outcome tools and follow-ups. Right column shows mean scores and standard deviations of each score. The right most column shows $P$ values obtained by comparing the 2 groups; significant differences are reported. Constant-Murley scores at 3-, 6-, and 12-month evaluations showed superiority for the augmented repair.

group A and $9.6 \mathrm{~kg}$ in group B; nevertheless, strength recovery was faster in the augmented group, and the difference with the nonaugmented group was statistically significant until the 1-year follow-up (Table IV).

During the entire study, only 2 patients reported complications, and in both cases the patients had a fall, causing rerupture of the tendon, requiring a biopsy during revision surgery $^{2}$ (Fig. 3).

\section{Discussion}

The primary endpoint of this single-center prospective randomized study was to demonstrate a better healing 
Table IV Secondary (radiologic) outcomes of the 2 groups

\begin{tabular}{llcr}
\hline & Group A & Group B & $P$ value \\
\hline Thickness, mm & & & \\
$\quad$ Baseline & $4.6(1.4)$ & $5.0(1.7)$ & .51 \\
1 mo & $6.0(1.5)$ & $4.4(1.8)$ & $<.01$ \\
6 mo & $5.0(1.5)$ & $3.9(1.7)$ & $<.01$ \\
12 mo & $4.6(1.4)$ & $3.7(1.1)$ & $<.01$ \\
24 mo & $4.4(1.3)$ & $3.6(1.1)$ & .01 \\
Footprint (H+T), mm & & & \\
1 mo & $9.4(3.2)$ & $7.5(4.1)$ & .06 \\
6 mo & $9.4(3.5)$ & $8.0(3.2)$ & .12 \\
12 mo & $9.4(3.0)$ & $8.4(3.0)$ & .21 \\
24 mo & $9.5(2.7)$ & $7.4(3.0)$ & $<.01$ \\
Density (H+T), points & & & \\
Baseline & $86.2(53.5)$ & $121.5(119.1)$ & .22 \\
1 mo & $82.1(92.8)$ & $112.5(67.5)$ & $<.01$ \\
6 mo & $86.8(38.6)$ & $112.4(60.6)$ & .09 \\
12 mo & $88.1(145.3)$ & $89.1(49.4)$ & .13 \\
24 mo & $87.3(143.7)$ & $88.4(53.7)$ & .77 \\
\hline
\end{tabular}

$H$, healed; $T$, thinned but healed.

Left column indicates MRI parameters (see the text for parameter explanation) and follow-ups. The 2 middle columns show mean scores and standard deviations (in parentheses) of each parameter. The rightmost column shows $P$ values obtained by comparing the 2 groups; significant differences are reported in bold. Group A showed a superior repair in terms of thickness, footprint coverage, and signal density. rate in the augmented group compared to a control group as verified by an MRI. At the 24-month follow-up, MRIs indicated a healing rate of $97.6 \%$ in the augmented group but only $59.5 \%$ in the nonaugmented group $(P<.001)$. These results are in line with those of the only similar study in the literature. ${ }^{32}$ The augmented group showed a minimum reduction in the percentage of healing during the first 6 months (Tables V and VI), which was expected and can probably be attributed to the remodeling and modifications that occurred during the rehabilitation period. The healing rate remained constant in the study group at the subsequent follow-ups; this was also expected and indirectly supports the beneficial effect of the remodeling process. Taking into account only the healed category we noted a slight reduction of the healing rate between the 12th and the 24th months follow-up, but no retear occurred during this period of time, meaning that the mean tendon thickness reduced between the 1 year and the 2 years follow-up.

The nonaugmented group showed a decrease in the healing rate over time, as well as a reduction of the mean tendon thickness (Table IV). Two patients had a positive outcome at 12 months to a retear at 24 months; on the other hand, 4 patients who were considered not healed until the 12th month were evaluated as healed or thinned but healed at the 24-month follow-up.

This can be an effect of a residual edema within the tendon that makes it appear as torn, increasing the signal intensity; the reabsorption of the edema during the following month revealed the continuity of the tendon at 24 months follow-up.

Secondary end points were a more physiologic tendon in terms of thickness, footprint coverage, and density. MRIs showed how the tendons in the augmented group were comparable to healthy tendons, whereas those of the control group had a less than optimal footprint coverage and a thinned tendon.

Moreover, the augmented group had a faster strength and functional recovery; even at 24 months, the 2 groups showed no difference in mean strength and mean Constant score.

There were no differences in functional patient-related scores throughout the entire follow-up period.

Only 2 complications were reported during the observation period, neither of them were related to the application of the scaffold itself. These occurrences allowed for histologic evaluations, providing evidence of scaffold incorporation and new tissue formation, showing typical characteristics of a tendon, and a functional remodeling according to load application, with crimps formation and a proper ultrastructure organization.

In the literature there is contrasting evidence regarding the patch augmentation in rotator cuff repair; Petri et $\mathrm{al}^{25}$ achieved results similar to ours, concluding that it is a safe and effective treatment even for patients with massive,

Table V Outcome comparison: healed category vs. other categories at each follow-up between the 2 groups

\begin{tabular}{|c|c|c|c|c|c|}
\hline \multirow[t]{2}{*}{ All } & \multicolumn{2}{|l|}{ Group A } & \multicolumn{2}{|l|}{ Group B } & \multirow[t]{2}{*}{$P$ value } \\
\hline & Healed & 0thers & Healed & Others & \\
\hline $1 \mathrm{mo}$ & $43(93.5)$ & $3(6.5)$ & $24(52.2)$ & $22(47.8)$ & $<.01$ \\
\hline $6 \mathrm{mo}$ & $38(82.6)$ & $8(17.4)$ & $19(41.3)$ & $27(58.7)$ & $<.01$ \\
\hline $12 \mathrm{mo}$ & $38(84.4)$ & $7(15.6)$ & $20(44.4)$ & $25(55.6)$ & $<.01$ \\
\hline $24 \mathrm{mo}$ & $32(78.0)$ & $9(19.5)$ & $9(24.3)$ & $28(75.7)$ & $<.01$ \\
\hline
\end{tabular}

Results are reported in terms of absolute values and percentages. A statistical improvement was noted for Group $A$ at each evaluation. 
Table VI Outcome comparison: healed $(H)$ and healed but thinned $(T)$ vs. other categories at each follow-up between groups

\begin{tabular}{|c|c|c|c|c|c|}
\hline \multirow[t]{2}{*}{ All } & \multicolumn{2}{|l|}{ Group A } & \multicolumn{2}{|l|}{ Group B } & \multirow[t]{2}{*}{$P$ value } \\
\hline & $\mathrm{H}+\mathrm{T}$ & 0thers & $\mathrm{H}+\mathrm{T}$ & Others & \\
\hline $1 \mathrm{mo}$ & $45(97.8)$ & $1(2.2)$ & $35(76.1)$ & $11(23.9)$ & $<.01$ \\
\hline $6 \mathrm{mo}$ & $44(95.7)$ & $2(4.3)$ & $31(67.4)$ & $15(32.6)$ & $<.01$ \\
\hline $12 \mathrm{mo}$ & $43(95.6)$ & $2(4.4)$ & $30(66.7)$ & $15(33.3)$ & $<.01$ \\
\hline $24 \mathrm{mo}$ & $40(97.6)$ & $1(2.4)$ & $22(59.5)$ & $15(40.5)$ & $<.01$ \\
\hline
\end{tabular}

Results are reported in terms of absolute values and percentage. A significant improvement can be noted for group $A$ at each evaluation.

retracted rotator cuff tears, providing a 9/10 satisfaction rate, with no complications or problems associated with the graft itself and significant improvements in all functionality scores. Other authors, though, reported worse outcomes when comparing a porcine dermal patch with other graft types. Steinhaus et $\mathrm{al}^{29}$ performed a systemic review comparing the outcome of synthetic scaffold, allograft and xenograft with the last reporting the worst outcome in terms or motion and of functional scores. Moreover, an overall retear rate of $25 \%$ and rates of $44 \%, 23 \%$, and $15 \%$ for xenografts, allografts, and synthetic grafts, respectively were reported. However, these studies are hardly comparable to the present one because of the different patches that were used, and because of the design of the study and its level of evidence.

The positive results in this study could have several explanations. From a biomechanical standpoint, Mihata et al conclude that suturing the tendon to the bone and reinforcing it by suturing the patch on top of the torn tendon at the musculotendinous junction and affixing it to the greater tuberosity create a "superior capsule reconstruction effect," 19 providing a restraint to the superior translation of the humeral head. Data from other groups also explain that this technique can reinforce and protect the repair reducing the stress over the tendon suture interface that is considered to be the weakest point of the repair. ${ }^{4}$ In a tensile testing to failure, Omae et $\mathrm{al}^{23}$ found that this augmentation technique increases the maximum load without increasing the stiffness of the repair. Jung et al ${ }^{16}$ demonstrated a $50 \%$ to $60 \%$ increase in the maximum force at failure after augmentation with a xenologous dermal patch, probably explained by the self-reinforcing effect demonstrated recently by Burkhart et al. ${ }^{5}$ Moreover, synthetic grafts seem to be less effective in protecting the repair, whereas collagen grafts may even resorb too quickly, ${ }^{9}$ Gore-Tex grafts showed high elongation values. ${ }^{18}$ Despite interesting results, some skepticism still remains because in both canine ${ }^{11}$ and human cadaveric ${ }^{7}$ models, it was demonstrated that compared with normal tendons, the repaired tendon was inferior.
From an ultrastructural standpoint, the porcine dermal patch demonstrated a proper integration with the adjacent tendon tissue at 24 months, an absence of macrophages and giant cells, and a mature tendon-bone insertion. These data appear superior compared with porcine subintestinal mucosa, which presents areas of calcification, fibrocartilage, and ectopic bone formation. ${ }^{22}$ Lastly, the noncrosslinked porcine dermal patches used in this study were shown to promote the expression of collagen type I and III in tenocytes, which are responsible for tendon strength, healing, and fibrosis. ${ }^{28}$

The main strength of this study lies in its design-a prospective randomized controlled trial with an appropriate sample size and a comprehensive assessment.

Nevertheless this study has some limitations. The follow-up at 24 months might be considered too short; however, a recent analysis of 221 consecutive repairs showed that nonhealing and retears occurred infrequently in the late postoperative period (after 3 months) in well-healed tendons that showed intact cuff repair with sufficient mechanical and biological healing. ${ }^{17}$ Moreover, after 24 months, the present study showed that patients treated by augmentation presented significant improvements with all the outcome tools administered, and could be considered healed by functional, radiographic, and histologic evidence. In addition, the repair site presented an appropriate regenerative tissue and structure, and the scaffold was completely integrated by 8 months. Another limitation was that a randomization able to equally distribute early and late retraction types in the 2 groups was not performed, and there was a slight prevalence of late retracted tendons in group A. To reduce evaluation biases, the obtained outcome was analyzed according to retraction types; the analysis still shows a better outcome for the augmented group compared to the control group.

In this study, we repaired tendons with the same technique in both groups; nevertheless, in the study group, the healing of the torn tendon could be favored by the fact that the scaffold is sutured from the myotendinous junction to the lateral anchor's row. This suture could reduce the load on the repaired tendon and thus favor healing at the 
tendon-to-bone interface, increasing the healing rate of the study group.

\section{Conclusion}

Rotator cuff repairs augmented with a porcine dermal patch result in excellent clinical outcomes. The technique is safe and effective, it is reproducible and allows better outcomes compared with those in standard medialized single-row repairs.

\section{Disclaimer}

The authors, their immediate families, and any research foundations with which they are affiliated have not received any financial payments or other benefits from any commercial entity related to the subject of this article.

\section{References}

1. Avanzi P. "Goal post technique" for arthroscopic biological augmentation of rotator cuff repair, VuMedi. https://www.vumedi. com/video/goal-post-technique-for-arthroscopic-biological-augmenta tion-of-rotator-cuff-repair/, accessed December 14, 2018

2. Avanzi P, Dei Giudici L, Gigante A, Zorzi C. How to manage failed rotator cuff repair: biologic augmentation. In: Milano G, Grasso A, Calvo A, Brzóska R, editors. Management of failed shoulder surgery; 2018. p. 219-28. Berlin: Springer.

3. Barber FA, Burns JP, Deutsch A, Labbé MR, Litchfield RB. A prospective, randomized evaluation of acellular human dermal matrix augmentation for arthroscopic rotator cuff repair. Arthroscopy 2012; 28:8-15. https://doi.org/10.1016/j.arthro.2011.06.038

4. Beitzel K, Chowaniec DM, McCarthy MB, Cote MP, Russell RP, Obopilwe E, et al. Stability of double-row rotator cuff repair is not adversely affected by scaffold interposition between tendon and bone. Am J Sports Med 2012;40:1148-54. https://doi.org/10.1177/ 0363546512437835

5. Burkhart SS, Adams CR, Burkhart SS, Schoolfield JD. A biomechanical comparison of 2 techniques of footprint reconstruction for rotator cuff repair: the SwiveLock-FiberChain construct versus standard double-row repair. Arthroscopy 2009;25:274-81. https://doi.org/ 10.1016/j.arthro.2008.09.024

6. Burkhart SS, Diaz Pagàn JL, Wirth MA, Athanasiou KA. Cyclic loading of anchor-based rotator cuff repairs: confirmation of the tension overload phenomenon and comparison of suture anchor fixation with transosseous fixation. Arthroscopy 1997;13:720-4.

7. Chaudhury S, Holland C, Thompson MS, Vollrath F, Carr AJ. Tensile and shear mechanical properties of rotator cuff repair patches. J Shoulder Elbow Surg 2012;21:1168-76. https://doi.org/10.1016/j.jse. 2011.08.045

8. Cho CH, Lee SM, Lee YK, Shin HK. Mini-open suture bridge repair with porcine dermal patch augmentation for massive rotator cuff tear: surgical technique and preliminary results. Clin Orthop Surg 2014;6: 329-35. https://doi.org/10.4055/cios.2014.6.3.329

9. Ciampi P, Scotti C, Nonis A, Vitali M, Di Serio C, Peretti GM, et al. The benefit of synthetic versus biological patch augmentation in the repair of posterosuperior massive rotator cuff tears: a 3-year follow-up study. Am J Sports Med 2014;42:1169-75. https://doi.org/10.1177/ 0363546514525592

10. Connor J, McQuillan D, Sandor M, Wan H, Lombardi J, Bachrach N, et al. Retention of structural and biochemical integrity in a biological mesh supports tissue remodeling in a primate abdominal wall model. Regen Med 2009;4:185-95. https://doi.org/ 10.2217/17460751.4.2.185

11. Derwin KA, Baker AR, Spragg RK, Leigh DR, Iannotti JP. Commercial extracellular matrix scaffolds for rotator cuff tendon repair Biomechanical, biochemical, and cellular properties. J Bone Joint Surg Am 2006;88:2665-72. https://doi.org/10.2106/JBJS.E.01307

12. Fuchs B, Weishaupt D, Zanetti M, Hodler J, Gerber C. Fatty degeneration of the muscles of the rotator cuff: assessment by computed tomography versus magnetic resonance imaging. J Shoulder Elbow Surg 1999;8:599-605.

13. Goutallier D, Postel JM, Bernageau J, Lavau L, Voisin MC. Fatty muscle degeneration in cuff ruptures. Pre- and postoperative evaluation by CT scan. Clin Orthop Relat Res 1994:78-83.

14. Hamada K, Fukuda H, Mikasa M, Kobayashi Y. Roentgenographic findings in massive rotator cuff tears. A long-term observation. Clin Orthop Relat Res 1990:92-6.

15. Jain NB, Higgins LD, Losina E, Collins J, Blazar PE, Katz JN Epidemiology of musculoskeletal upper extremity ambulatory surgery in the United States. BMC Musculoskelet Disord 2014;15:4. https:// doi.org/10.1186/1471-2474-15-4

16. Jung C, Spreiter G, Audigé L, Ferguson SJ, Flury M. Patch-augmented rotator cuff repair: influence of the patch fixation technique on primary biomechanical stability. Arch Orthop Trauma Surg 2016;136:609-16. https://doi.org/10.1007/s00402-016-2436-6

17. Kim JH, Hong IT, Ryu KJ, Bong ST, Lee YS, Kim JH. Retear rate in the late postoperative period after arthroscopic rotator cuff repair. Am J Sports Med 2014;42:2606-13. https://doi.org/10.1177/ 0363546514547177

18. McKeown AD, Beattie RF, Murrell GA, Lam PH. Biomechanical comparison of expanded polytetrafluoroethylene (ePTFE) and PTFE interpositional patches and direct tendon-to-bone repair for massive rotator cuff tears in an ovine model. Shoulder Elbow 2016;8:22-31. https://doi.org/10.1177/1758573215601815

19. Mihata T, McGarry MH, Pirolo JM, Kinoshita M, Lee TQ. Superior capsule reconstruction to restore superior stability in irreparable rotator cuff tears: a biomechanical cadaveric study. Am J Sports Med 2012;40:2248-55. https://doi.org/10.1177/0363546512456195

20. Mikos AG, McIntire LV, Anderson JM, Babensee JE. Host response to tissue engineered devices. Adv Drug Deliv Rev 1998;33:111-39.

21. Neri BR, Chan KW, Kwon YW. Management of massive and irreparable rotator cuff tears. J Shoulder Elbow Surg 2009;18:808-18. https://doi.org/10.1016/j.jse.2009.03.013

22. Nicholson GP, Breur GJ, Van Sickle D, Yao JQ, Kim J, Blanchard CR. Evaluation of a cross-linked acellular porcine dermal patch for rotator cuff repair augmentation in an ovine model. J Shoulder Elbow Surg 2007;16(Suppl):S184-90. https://doi.org/10.1016/j.jse.2007.03.010

23. Omae H, Steinmann SP, Zhao C, Zobitz ME, Wongtriratanachai P, Sperling JW, et al. Biomechanical effect of rotator cuff augmentation with an acellular dermal matrix graft: a cadaver study. Clin Biomech (Bristol Avon) 2012;27:789-92. https://doi.org/10.1016/j.clinbiomech. 2012.05.001

24. Patte D. Classification of rotator cuff lesions. Clin Orthop Relat Res 1990:81-6.

25. Petri M, Warth RJ, Horan MP, Greenspoon JA, Millett PJ. Outcomes after open revision repair of massive rotator cuff tears with biologic patch augmentation. Arthroscopy 2016;32:1752-60. https://doi.org/10. 1016/j.arthro.2016.01.037

26. Ricchetti ET, Aurora A, Iannotti JP, Derwin KA. Scaffold devices for rotator cuff repair. J Shoulder Elbow Surg 2012;21:251-65. https://doi. org/10.1016/j.jse.2011.10.003

27. Shea KP, Obopilwe E, Sperling JW, Iannotti JP. A biomechanical analysis of gap formation and failure mechanics of a xenograft-reinforced 
rotator cuff repair in a cadaveric model. J Shoulder Elbow Surg 2012;21: 1072-9. https://doi.org/10.1016/j.jse.2011.07.024

28. Smith RDJ, Carr A, Dakin SG, Snelling SJB, Yapp C, Hakimi O. The response of tenocytes to commercial scaffolds used for rotator cuff repair. Eur Cells Mater 2016;31:107-18. https://doi.org/10.22203/ eCM.v031a08

29. Steinhaus ME, Makhni EC, Cole BJ, Romeo AA, Verma NN. Outcomes after patch use in rotator cuff repair. Arthroscopy 2016;32: 1676-90. https://doi.org/10.1016/j.arthro.2016.02.009

30. Tuoheti Y, Itoi E, Yamamoto N, Seki N, Abe H, Minagawa H, et al. Contact area, contact pressure, and pressure patterns of the tendon-bone interface after rotator cuff repair. Am J Sports Med 2005;33:1869-74. https://doi.org/10.1177/ 0363546505278256
31. Xu H, Wan H, Zuo W, Sun W, Owens RT, Harper JR, et al. A porcinederived acellular dermal scaffold that supports soft tissue regeneration: removal of terminal galactose-a-(1,3)-galactose and retention of matrix structure. Tissue Eng Part A 2009;15:1807-19. https://doi.org/10. 1089/ten.tea.2008.0384

32. Yamaguchi K, Ditsios K, Middleton WD, Hildebolt CF, Galatz LM, Teefey SA. The demographic and morphological features of rotator cuff disease: a comparison of asymptomatic and symptomatic shoulders. J Bone Joint Surg Am 2006;88:1699-704. https://doi.org/10. 2106/JBJS.E.00835

33. Yoo JC, Ahn JH, Koh KH, Lim KS. Rotator cuff integrity after arthroscopic repair for large tears with less-than-optimal footprint coverage. Arthroscopy 2009;25:1093-100. https://doi.org/10.1016/j. arthro.2009.07.010 